\title{
sciendo
}

POLISH MARITIME RESEARCH 2 (98) 2018 Vol. 25; pp. 37-46

10.2478/pomr-2018-0052

\section{PROPAGATION OF SHIP-GENERATED NOISE IN SHALLOW SEA}

\author{
Eugeniusz Kozaczka ${ }^{1,2}$ \\ Grażyna Grelowska ${ }^{1}$ \\ ${ }^{1}$ Gdańsk University of Technology, Poland \\ ${ }^{2}$ Polish Naval Academy, Poland
}

\begin{abstract}
Contamination of sea environment by noise and any energy radiated to water constitutes today a problem to which more and more attention is paid, in view, a.o., of consequences of an impact of these factors onto marine fauna. European Union has introduced a directive by which EU countries are made responsible to undertake efforts aimed at reaching a good envirenmental status of European seas by 2020. A main source of underwater noise is sea transport of any kind. Propagation of underwater acoustic disturbances in the Baltic Sea highly differs from the course of the phenomenon in a deep sea. Model of spherical propagation cannot be applied to this case in view of water environment limitation by seabed and free water surface, i.e. a reduction of the problem to wave propagation in a water layer of the depth comparable with acoustic wave length. This paper is aimed at demonstration of possible assessment of range of acoustic disturbances generated by a ship sailing in shallow sea, by using a method described in the work [13]. The research was made on the basis of results of own measurements of underwater noise produced by ships in the Gdansk Bay area. An important factor which decIdes on a range of underwater disturbances is a kind of seabed sediments. In this paper there are presented results of numerical investigations based on real data dealing with noise produced by a selected floating unit (ship) for selected characteristic spectral components. The simulations were conducted for the shallow sea model of definite physical parameters such as acoustic wave propagation velocity, geometrical dimensions (water depth) and seabed acoustic parameters as well.
\end{abstract}

Keywords: shallow water acoustics, propagation of acoustic waves in a water layer, wave modes in shallow sea, underwater noise produced by ship

\section{INTRODUCTION}

Current conditions of an acoustic underwater climate are mainly associated with ship traffic over a given sea area. In this paper there are described consequences of underwater noise generation by moving ships and other objects such as recreation fast motor craft.

It's common knowledge that the underwater environment contamination by noise has been growing year by year especially close to fairways along which transport ships, touristic motor boats and other water surface communication means operate [16], but also close to active objects of marine infrastructure [4]. The situation becomes relatively serious as excessive noise and other energy sources significantly affect not only living conditions inside ship [20] but also underwater biological life $[1,15]$ which becomes more and more endangered not only by noise, light, uncontrolled impact of chemical products, but also unbalanced management of fish resouces.

For thts reason the EU has issued an appropriate directive obligating EU countries to obey definite acoustic standards concerning water environment. The directive of EU Parliament and Council 2008/56/WE of 17 June 2008 enacts framework for EU activities in the domain of sea environmental policy, within which EU countries have to undertake necessary actions aimed at reaching or maintaining a good environmental status of sea waters (Good Environmental Status - GES) not later than before $2020[3,18]$. According to the directive, in order to protect sea water environment, EU countries have to prepare 
and implement a maritime strategy covering a.o.: the working out of a preliminary assessment of sea water environmental status, a set of typical features of good environmental status, a set of environmental aims, and working out and implementing a sea water monitoring program.

In the directive 2008/56/WE there are defined 11 descriptive indices for which an assessment as to the defined criteria of good environmental status should be performed. One of them is the index W11 - Underwater noise and other energy sources.

It's common knowledge that size of the ships grows along with technical progress. Hence, power of devices installed on them, especially of propulsion systems significantly increases. This results in significant rise of noise associated with operation of ship propellers as well as respective vibration energy transmitted to water environment.

Relating the considerations to the Baltic Sea, especially to its southern region, one can state that propagation of acoustic waves is of a specific character associated with small depth of the water areas in question $[7,8]$. In view of occurrence of the geometrical limitations of the medium in which acoustic wave propagates, namely, close presence of free water surface and seabed, a form of propagation of disturbances definitely differs from that typical for deep waters $[2,10,11]$ where water depth is much greater than length of wave of the lowest frequency produced by a ship sailing nearby.

Therefore in a distance from the ship there is produced an acoustic field which has a character of moving waves, called wave modes. This results from a complex structure of interferring waves which multifold reflect from both seabed surface and free sea water surface $[14,17]$.

Apart from the phenomenon of reflection from free sea water surface we have to do with the change in wave phase by the angle $\pi$, which means that the wave reflected from the surface is in counter-phase in regard to the projecting wave. Therefore the interferential acoustic field has a property of moving the wave modes, for which a group velocity is characteristic. It means that we have to do with the phenomenon of geometrical dispersion of velocity, known fom physics.

Apart from the modal propagation of acoustic waves, additional phenomena associated with presence of seabed take place. The seabed has physical features called the material constants such as density and elastic wave propagation velocity $[9,19]$, of values close to respective properties of sea water. It means that seabed impedance is usually greater or a little greater than that of sea water. For this reason some part of acoustic wave energy penetrates the seabed. In case of a rocky seabed, i.e. that having shape elasticity, additional transverse acoustic waves in the range associated with a spectrum of acoustic waves produced by underwater acoustic disturbance sources (i.e. ships), may occur.

In this paper there are presented results of numerical investigations based on the real data from measurements of noise produced by a selected floating unit (ship) [6], concerning selected characteristic spectral components. The simulations were performed by applying a shallow sea model of definite physical parameters such as acoustic wave propagation velocity, geometrical dimensions (water depth) as well as acoustic parameters of seabed, that was discussed more thoroughly in the publications $[13,14]$.

The data in question were presented in the form of distribution of particular wave modes in function of water depth for selected horizontal distances from source of disturbances. Results of the investigations will be used for the determining of acoustic climate of a water area, that is the key procedure for taking into account a single object emitting underwater noise.

\section{ANALYSIS OF ACOUSTIC FIELD DISTRIBUTION IN A LIQUID LAYER BY USING WAVE METHOD}

Let's consider an acoustic pressure field resulting from a point source of a spherical wave in an infinite, flat-parallel water layer which has to model a shallow sea. Fig. 1 shows a schematic diagram of a perfectly flat acoustic system.

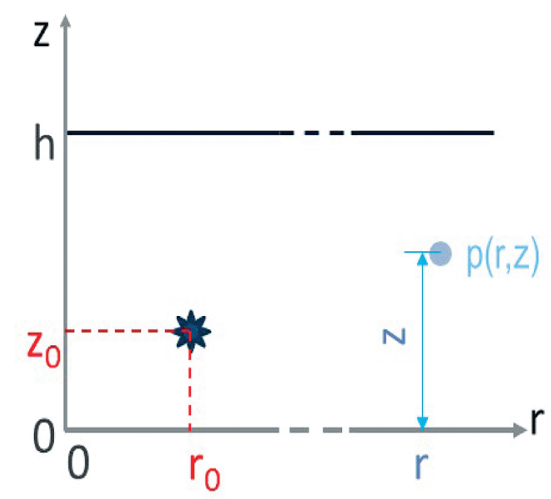

Fig. 1. Cross-section of a perfectly flat acoustic system

The distribution of acoustic pressure generated by a source we determine by solving the wave equation in the threedimensional orthogonal Cartesian space.

$$
\nabla^{2} \bar{p}(x, y, z, t)-\frac{1}{c^{2}} \frac{\partial^{2} \bar{p}(x, y, z, t)}{\partial t^{2}}=\bar{f}(x, y, z, t)
$$

$$
\begin{aligned}
& \left.\bar{p}(x, y, z, t)\right|_{t=0}=h(x, y, z) \\
& \left.\frac{\partial \bar{p}(x, y, z, t)}{\partial t}\right|_{t=0}=g(x, y, z)
\end{aligned}
$$

for

$$
\left\{\begin{array}{c}
-\infty<x<\infty \\
-\infty<y<\infty \\
0 \leq z \leq h \\
0<t<\infty
\end{array}\right.
$$

for $\quad\left\{\begin{array}{c}-\infty<x<\infty \\ -\infty<y<\infty \\ 0 \leq z \leq h\end{array}\right.$ 
and the boundary conditions as follows:

$$
\begin{array}{ll}
{\left.\left[\frac{\partial \bar{p}(x, y, z, t)}{\partial z}+\sigma_{1} \bar{p}(x, y, z, t)\right]\right|_{z=0}=0} & \text { for } \quad\left\{\begin{array}{l}
-\infty<x<\infty \\
-\infty<y<\infty
\end{array}\right. \\
{\left[\frac{\partial \bar{p}(x, y, z, t)}{\partial z}+\left.\sigma_{2} \bar{p}(x, y, z, t)\right|_{z=h}=0\right.} &
\end{array}
$$

The limiting conditions of the problem (1) in the form (2) and (3) were formulated in a general way.

We will consider a definite case putting: $\bar{p}(x, y, z, t)=p(x, y, z) e^{-i \omega t}$ at the assumption that the planes $z=0$ and $z=h$ are perfectly reflecting. Putting $\sigma_{1}=0$ for $z<0$ we reach, for $z<0$, a perfectly rigid half-space.

Whereas putting $\sigma_{1}=\infty$ for $z>h$ we get a perfectly soft half-space.

Introducing the above mentioned relations into the equation (1) and the limiting conditions (2) and (3) we obtain:

$$
\begin{aligned}
& \nabla^{2} p(x, y, z)+k^{2} p(x, y, z)=f(x, y, z) \quad \text { for } \quad\left\{\begin{array}{c}
-\infty<x<\infty \\
-\infty<y<\infty \\
0 \leq z \leq h
\end{array}\right. \\
& \begin{array}{ll}
\left.\frac{\partial p(x, y, z)}{\partial z}\right|_{z=0}=0 & \text { for } \quad\left\{\begin{array}{l}
-\infty<x<\infty \\
-\left.\infty(x, y, z)\right|_{z=h}=0
\end{array}\right.
\end{array}
\end{aligned}
$$

Using the horizontal symmetry of the problem we introduce the cylindrical reference frame:

$$
\begin{array}{ccc}
\nabla^{2} p(r, z)+k^{2} p(r, z)=f(r, z) & \text { for } & \left\{\begin{array}{l}
0<r<\infty \\
0 \leq z \leq h
\end{array}\right. \\
\left.\frac{\partial p(r, z)}{\partial z}\right|_{z=0}=0 & \text { for } & 0<r<\infty \\
\left.p(r, z)\right|_{z=h}=0 & &
\end{array}
$$

and:

$$
f(r, z)=\frac{\rho \omega Q}{r} \delta(r) \delta\left(z-z_{0}\right)
$$

where:

$$
\nabla^{2} p(r, z)=\frac{1}{r} \frac{\partial}{\partial r}\left(r \frac{\partial p(r, z)}{\partial r}\right)+\frac{\partial^{2} p(r, z)}{\partial z^{2}}
$$

Where: $Q$ stands for source volumetric velocity, $\rho$ - density of a medium in which wave propagates, $\omega=2 \pi f$ - angular frequency. Applying the variables separation method we solve the problem (6) at the limiting conditions (7) and using the Sommerfeld radiation condition in the form:

$$
\lim _{r \rightarrow \infty} \sqrt{r}\left[\frac{\partial p r, z}{\partial r}-i k_{r} p(r, z)\right]=0
$$

The solution is obtained in the following form :

$$
p(r, z)=\frac{2 \pi \rho Q}{h} \sum_{n=0}^{\infty} \cos \frac{\pi}{h}\left(n+\frac{1}{2}\right) 2 \cos \frac{\pi}{h}\left(n+\frac{1}{2}\right) z_{0} H_{0}{ }^{(1)}\left(k_{r n} \cdot r\right)
$$

where: $\quad k_{r n}=\sqrt{1-\left[\frac{c}{\omega} \frac{\pi}{h}\left(n+\frac{1}{2}\right)\right]^{2}}$ - horizontal wave number, $\mathrm{H}_{0}{ }^{(1)}$ - Hankel functions of $1^{\text {st }}$ order.

Using asymptotic representation of Hankel function [2] in the form: $\quad \lim _{r \rightarrow \infty} H_{0}^{(1)}\left(k_{r n} r\right)=\sqrt{\frac{2}{\pi k_{r n} r}} e^{i\left(k_{r n} r+\frac{\pi}{4}\right)}$

we are able to write (10) in the following form:

$$
p(r, z)=\frac{2 \pi \rho Q}{h} \cdot e^{i \pi / 4} \sum_{n=0}^{\infty} \cos \frac{\pi}{h}\left(n+\frac{1}{2}\right) z \cos \frac{\pi}{h}\left(n+\frac{1}{2}\right) z_{0} \sqrt{\frac{2}{\pi k_{r n} r}} e^{i k_{r n} r}
$$


The relation (12) is of a summation form - it is consisted of wave modes moving along the layer. The pressure distribution of the first four wave modes in function of liquid depth is presented in Fig. 2.

The acoustic pressure distribution in function of depth behaves like a standing wave. The wave modes propagate along the layer with a different group velocity determined as follows :

$$
u_{n}=\frac{d \omega}{d k_{r n}}=c \sqrt{1-\left[\frac{c}{\omega} \frac{\pi}{h}\left(n+\frac{1}{2}\right)\right]^{2}}
$$

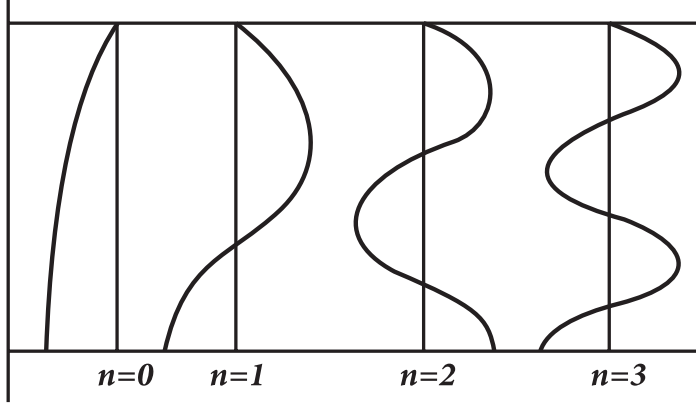

Fig. 2. Pressure distribution in transverse cross-section of liquid layer, for wave modes of the order $n=0,1,2,3$

If we take into account an impact of seabed of a definite impedance, we get the relation which describes the pressure distribution in the form (14):

$$
p(r, z)=\frac{2 \pi \rho Q}{h} \cdot e^{-i \pi / 4} \sum_{n=0}^{\infty} \cos \frac{\pi}{h}\left(n+\frac{1}{2}\right) z_{o} \cos \frac{\pi}{h}\left(n+\frac{1}{2}\right) z \sqrt{\frac{2}{\pi k_{r n} r}} e^{i k_{r n} r} e^{-\delta_{2 n} r}
$$

where:

$$
\delta_{2 n}=\frac{-\pi\left(n+\frac{1}{2}\right)}{h} \frac{\ln \left|b\left(\alpha_{n}^{0}\right)\right|}{2 \sqrt{(h k)^{2}-\left[\pi\left(n+\frac{1}{2}\right)\right]^{2}}}
$$

$$
\begin{array}{r}
b(\theta)=\frac{m \cos \theta-\sqrt{n_{1}^{2}-\sin ^{2} \theta}}{m \cos \theta+\sqrt{n_{1}^{2}-\sin ^{2} \theta}} \\
\alpha_{n}^{0}=\arcsin \frac{\pi\left(n+\frac{1}{2}\right)}{k h} .
\end{array}
$$

The relation (14) constitutes a simple representation of wave modes in function of layer depth. Compared with the relation (12) it contains additional factors which represent decreasing acoustic pressure with growing distance. A real value of the reflection coefficient $b$ responsible for the damping of particular wave modes is comprised in the exponent.

The above obtained relations were used to investigate a character of propagation of underwater disturbances produced by ships sailing over a shallow sea, for different kinds of seabed.

\section{CHARACTERISTICS OF UNDERWATER NOISE EMITTED BY SHIPS}

Ship traffic is a main source of underwater noise, especially in regions of fairways. Ship is a broad-band source of spectral characteristics which is tightly associated with applied design solutions as well as current working parameters of shipboard devices.

On ships, their propellers are the most efficient source of underwater noise. One of its parts is the propeller rotation frequency and its harmonics. Noise components coming from the propeller constitute usually a dominating part of underwater disturbances in the low-frequency band at large speeds of ship, especially when the propeller is in developed cavitation state [11]. Ship propulsion engine is the main source of underwater noise at a moderate speed of ship. Generally, a level of underwater noise spectrum components of a ship is not stable due to changes in propeller load in different sea states. The acoustic power radiated at the fundamental ignition frequency is associated with engine output and may be estimated equal to about $0,1 \%$ of the total engine power.
Information on spectrum characteristics, called also ship's signatures, we obtain on the basis of underwater noise measurements carried out in mobile or stationary testing grounds $[6,10]$. The mobile testing ground may be consisted of a few hydrophones fastened on a frame in some distance from each other. The measurements can be conducted with hydrophones arranged either in horizontal or vertical plane. A merit of such measuring system is its possible location in an arbitrary point of water area.

More precise measurements of underwater noise are conducted in stationary testing grounds. In this case sensors are located in strictly determined points and strictly determined depth whereas a tested ship moves over them at a given course. A drawback of the solution is that there is not possible to obtain results for one depth and one kind of seabed.

On the basis of measurement results a series of ship underwater noise characteristics can be obtained, that allows to assess pressure level changes in function of distance and depth for different ship speed values and different settings of shipboard devices. One of the characteristics is spectrum characteristics, called often ship's signature. An example ship's signature is shown in Fig. 3. 


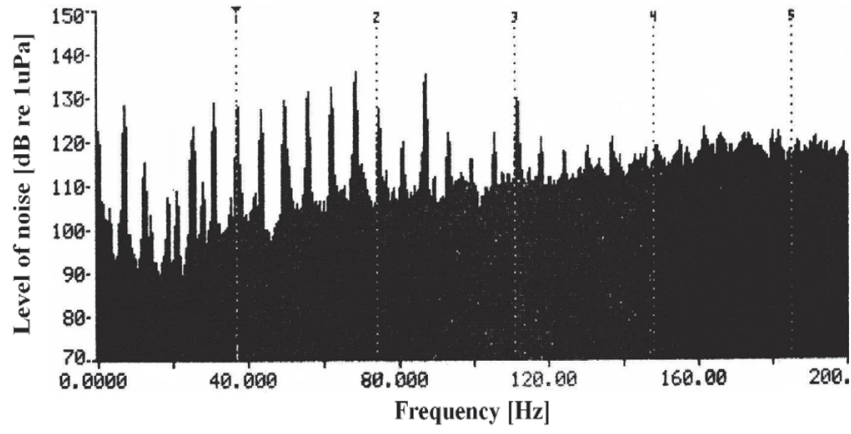

Fig. 3. Example ship's signature determined on the basis of underwater noise measurements conducted in the region of Gdansk Bay

Worth stressing, that, independent of ship's type, the main part of acoustic energy radiated to water is located within low frequency band, up to a few hundred $\mathrm{Hz}$.

\section{WAVEGUIDE SOUND PROPAGATION IN SHALLOW SEA}

Characteristic features of waveguide propagation in shallow sea will be presented on the example of results of experimental and numerical investigations of a tug. In the spectrum there is possible to distinguish a series of characteristic components within a rather broad frequency band, namely almost up to $1000 \mathrm{~Hz}$. In Fig. 4 are shown the components determined on the basis of measurements conducted under the ship in a $20 \mathrm{~m}$ deep water area with the use of a hydrophone placed $1 \mathrm{~m}$ over the seabed, whose pressure level exceeded $155 \mathrm{~dB}$.

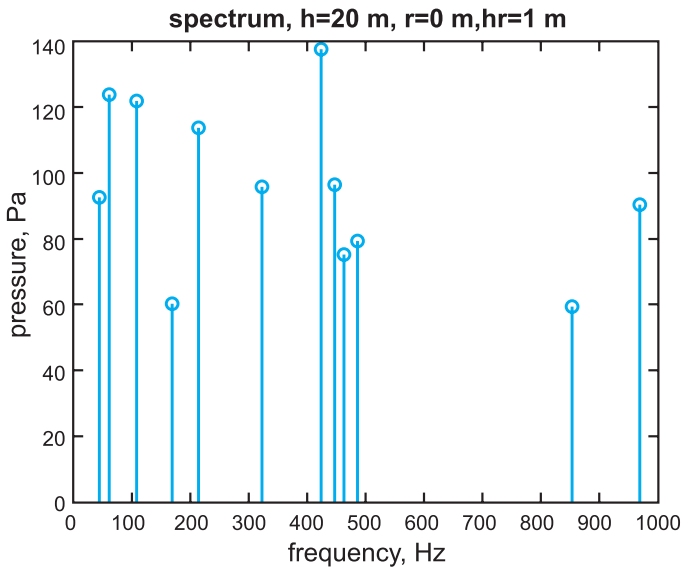

Fig. 4. Components of underwater noise spectrum of a tug, whose pressure level exceeded $155 \mathrm{~dB}$, i.e. about $56 \mathrm{~Pa}$

The calculations were made for all distinguished frequencies under the following assumptions:

- Water depth $\mathrm{h}=20 \mathrm{~m}$

- Salinity S=7.1 PSU

- Water temperature $\mathrm{T}=14^{\circ} \mathrm{C}$

- Sound speed in water, $\mathrm{c}=1470.8 \mathrm{~m} / \mathrm{s}$

- Water density, $\rho=1004.7 \mathrm{~kg} / \mathrm{m}^{3}$

- Source immersion depth $\mathrm{z}=1 \mathrm{~m}$ under water surface

There were considered situations of sound propagation over a sand, gravel and silt seabed, respectively, of the following geo-acoustic parameters $[9,19,5,12]$ :
- Medium silt: $\mathrm{m}=\rho_{1} / \rho=1.147 ; \mathrm{n}=\mathrm{c}_{1} / \mathrm{c}=0.9801$

- Fine sand: $m=\rho_{1} / \rho=1.2236 ; n=c_{1} / c=1.0364$

- Fine gravel: $m=\rho_{1} / \rho=2.4923 ; n=c_{1} / c=1.338$

Distributions of the total underwater noise of the tug within the frequency band up to $1000 \mathrm{~Hz}$ in a small distance from the ship, up to $500 \mathrm{~m}$, in the case of disturbance propagation over different types of seabed: fine sand, fine gravel and medium silt, are presented in Fig. 5, 6 and 7, respectively. It should be observed that the spatial pressure distribution depends on a type of seabed. The non-uniform pressure distribution in function of water depth results from the forming and propagating of wave modes.

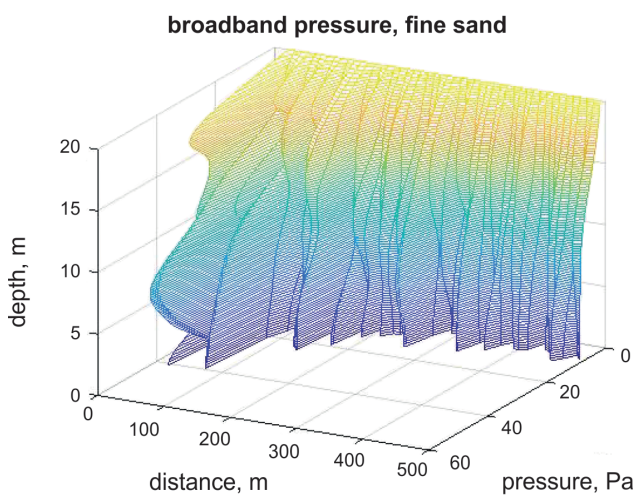

Fig. 5. Spatial distribution of noise produced by a tug sailing over fine sand seabed

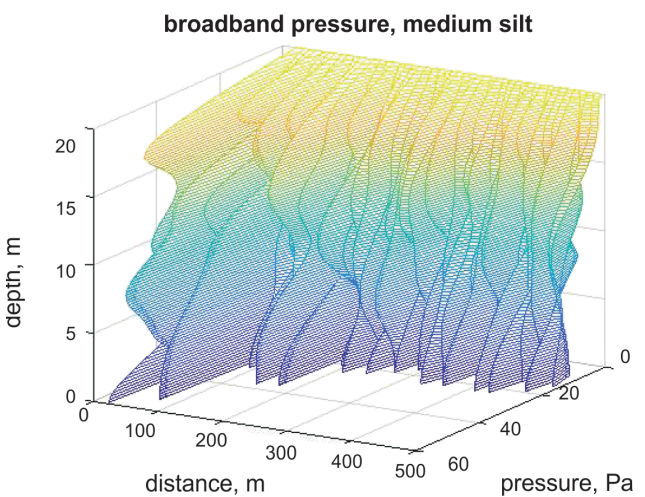

Fig. 6. Spatial distribution of noise produced by a tug sailing over medium silt seabed

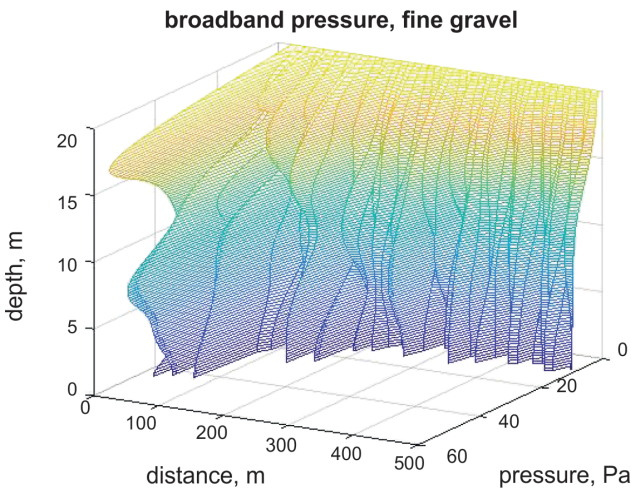

Fig. 7. Spatial distribution of noise produced by a tug sailing over fine gravel seabed 
Based on the subsequent diagrams, there is possible to examine an impact of formation of wave modes on distribution of resultant acoustic pressure for particular components of frequency spectrum of the tug in question. On the diagrams, are shown the vertical wave modes pressure distributions for the tug's spectrum components of $213 \mathrm{~Hz}, 426 \mathrm{~Hz}$ and $966 \mathrm{~Hz}$ frequency, respectively.
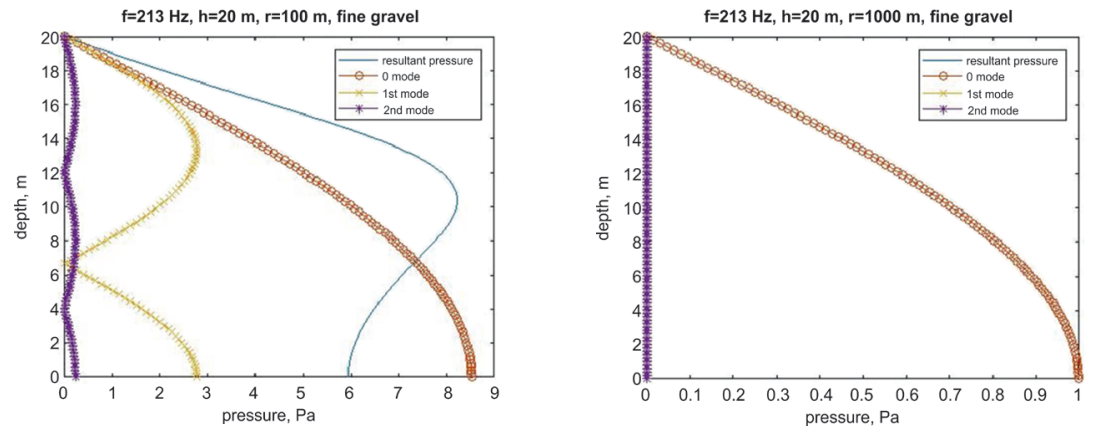

Fig. 8. Modes of the spectrum component of $213 \mathrm{~Hz}$ frequency in the distance of $100 \mathrm{~m}$ and $1000 \mathrm{~m}$ from the ship, respectively
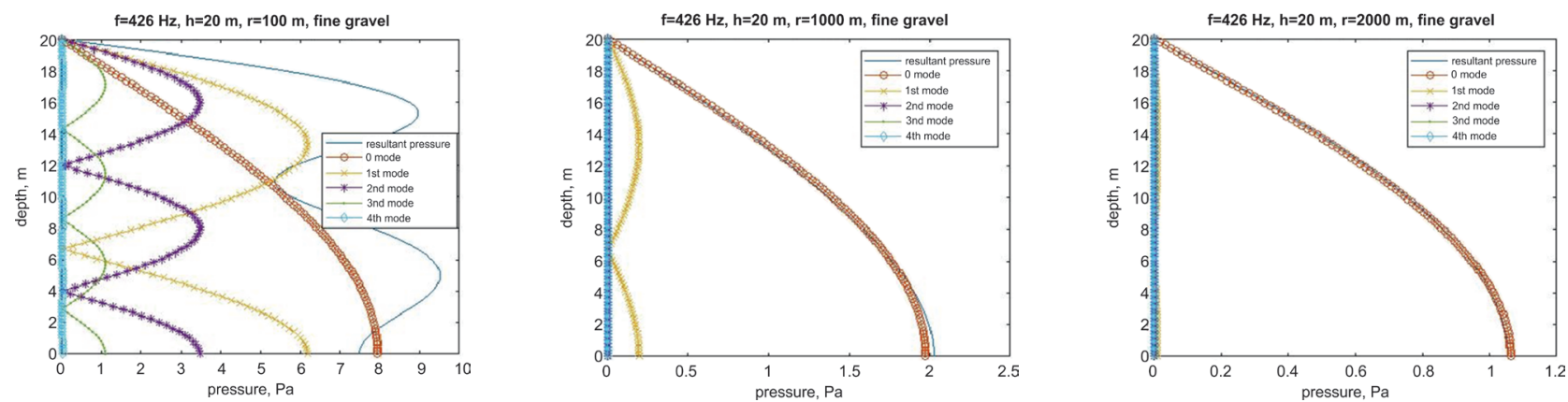

Fig. 9. Modes of the spectrum component of $426 \mathrm{~Hz}$ frequency in the distance of $100 \mathrm{~m}, 1000 \mathrm{~m}$ and $2000 \mathrm{~m}$ from the ship, respectively
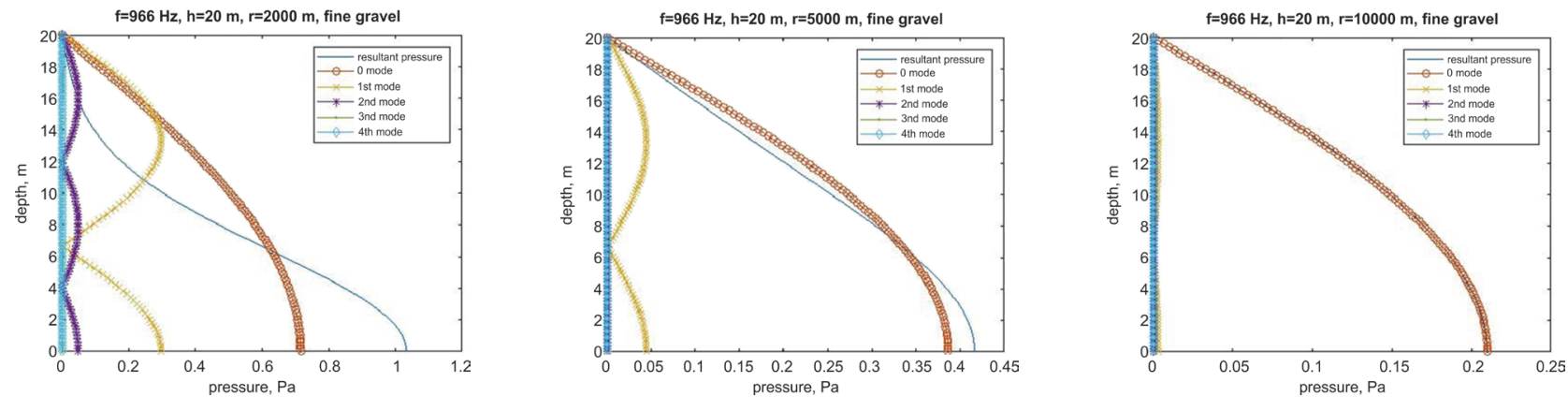

Fig. 10. Modes of the spectrum component of $966 \mathrm{~Hz}$ frequency in the distance of $2000 \mathrm{~m}, 5000 \mathrm{~m}$ and $10000 \mathrm{~m}$ from the ship, respectively

The wave modes start to form in a rather small distance from source, namely that at which spherical propagation resulting from reflections from the surfaces limiting the layer becomes conversed into cylindrical one. In the case in question the wave modes are formed for all components of the tug's spectrum, and their number depends on frequency. The higher the frequency the bigger the number of wave modes.

In the case of modes propagation we have to do with dispersion of sound propagation velocity. Every wave mode propagates with a different velocity. Tab. 1 shows values of propagation velocity of particular modes for the above considered spectral components.

The modes gradually fade out along with growing distance and finally we have propagation of a single mode. The distance in which modes of a higher order are faded out depends also on frequency. For instance, the acoustic wave of $213 \mathrm{~Hz}$ frequency is composed, in the distance of $100 \mathrm{~m}$ from the ship, of the modes of $0^{\text {th }}, 1^{\text {st }}$ and $2^{\text {nd }}$ order. In the distance

Tab. 1. Propagation velocity of particular modes for the selected components of the tug's frequency spectrum; the acoustic wave velocity in water $c=1470.8 \mathrm{~m} / \mathrm{s}$

\begin{tabular}{|c|c|c|c|c|c|}
\hline $\mathrm{f}[\mathrm{Hz}]$ & $\mathrm{n}=0$ & $\mathrm{n}=1$ & $\mathrm{n}=2$ & $\mathrm{n}=3$ & $\mathrm{n}=5$ \\
\hline 213,00 & 1470,793 & 1470,778 & 1470,763 & 1470,7478 & 1470,733 \\
\hline 426,00 & 1470,798 & 1470,794 & 1470,791 & 1470,787 & 1470,783 \\
\hline 966,00 & 1470,8 & 1470,799 & 1470,718 & 1470,7975 & 1470,797 \\
\hline
\end{tabular}


of $1000 \mathrm{~m}$ it takes the form of a single, $0^{\text {th }}-$ order mode. The acoustic wave component of $426 \mathrm{~Hz}$ frequency forms, in the neighbourhood of its source, the modes of $0^{\text {th }}, 1^{\text {st }}$, $2^{\text {nd }}$ and $3^{\text {rd }}$ order, in the distance of $1000 \mathrm{~m}$ the resultant pressure of the component is a sum of two modes, while in the distance of $2000 \mathrm{~m}$ the component propagates in the form of $0^{\text {th }}$ - order mode. The number of modes of the acoustic wave of $966 \mathrm{~Hz}$ frequency is even greater and the range of multi-mode propagation significantly exceeds the ranges for the lower frequencies. In this case, in the distance of $2000 \mathrm{~m}$ three modes: of $0^{\text {th }}, 1^{\text {st }}$, and $2^{\text {nd }}$ order are still visible, and the two highest - order modes Fig. 10. Modes of the spectrum component of $966 \mathrm{~Hz}$ frequency in the distance of $2000 \mathrm{~m}$, $5000 \mathrm{~m}$ and $10000 \mathrm{~m}$ from the ship, respectivelyalso in the distance of $5000 \mathrm{~m}$. In the distance of $10000 \mathrm{~m}$ we have again a single-mode propagation.

As observed, the range of the disturbance propagation in the form of wave modes increases along with increasing frequency of a spectrum component.

The assessment of impact of a kind of seabed on acoustic energy absorption we conduct on the basis of changes in the resultant pressure which is a sum of modes for a selected component of the tug's frequency spectrum. In Fig. 11, 12 and 13 there is demonstrated in which way the vertical resultantpressure distribution for the $213 \mathrm{~Hz}$ frequency component changes at different distances from the ship and under the assumption that we have to do with the seabed formed of fine gravel, fine sand and medium silt, respectively.

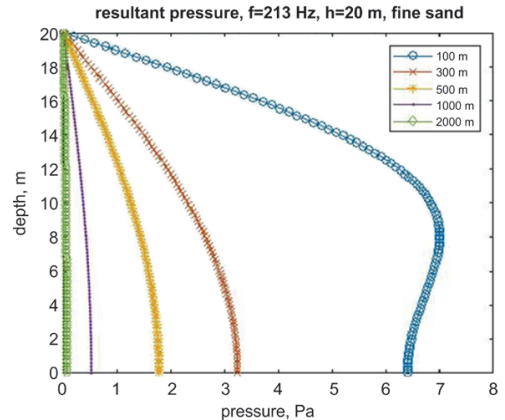

Fig.11. Vertical resultant-pressure distribution for the $213 \mathrm{~Hz}$ frequency spectrum component in different distances from the ship, in case of fine sand seabed

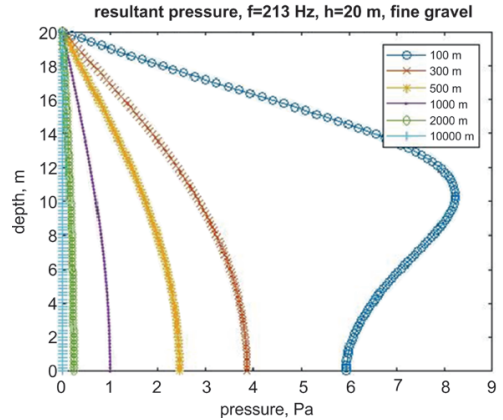

Fig. 12. Vertical resultant-pressure distribution for the $213 \mathrm{~Hz}$ frequency spectrum component in different distances from the ship, in case of fine gravel seabed.

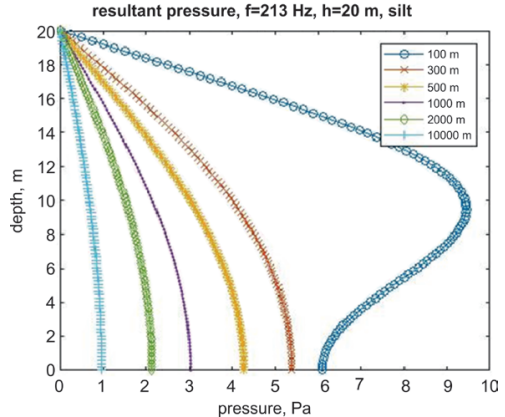

Fig. 13. Vertical resultant-pressure distribution for the $213 \mathrm{~Hz}$ frequency spectrum component in different distances from the ship, in case of medium silt seabed

The $213 \mathrm{~Hz}$ frequency component reaches $1000 \mathrm{~m}$ distance over the fine sand seabed, up to $2000 \mathrm{~m}$ - over the fine gravel seabed, and more than $10000 \mathrm{~m}$ - over the medium silt seabed.

12 significant frequency components were distinguished within the tug's spectrum. The acoustic energy emitted to water is composed mainly of them. Examining the subsequent diagrams we are able to assess the changes in vertical distributions of total tug-generated pressure, which occur along with growing distance. We may also assess impact of a kind of seabed on disturbance propagation range.

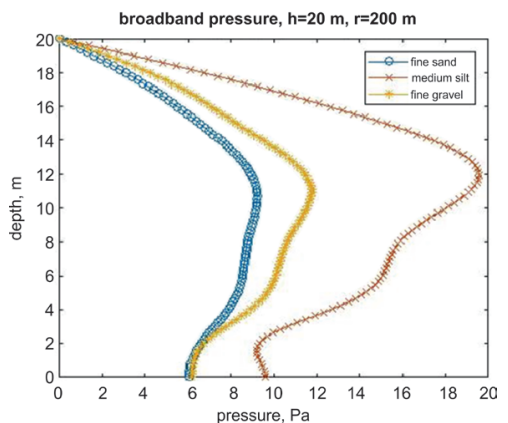

Fig. 14. Vertical distribution of total tug-generated pressure in the distance of $200 \mathrm{~m}$ from the ship in the case of propagation over the seabed composed of fine sand, medium silt and fine gravel, respectively

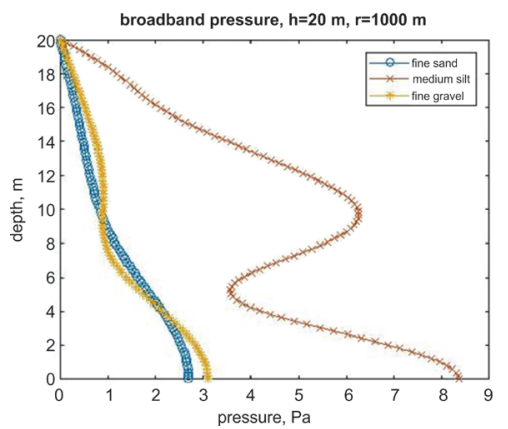

Fig. 15. Vertical distribution of total tug-generated pressure in the distance of $1000 \mathrm{~m}$ from the ship in the case of propagation over the seabed composed of fine sand, medium silt and fine gravel, respectively 


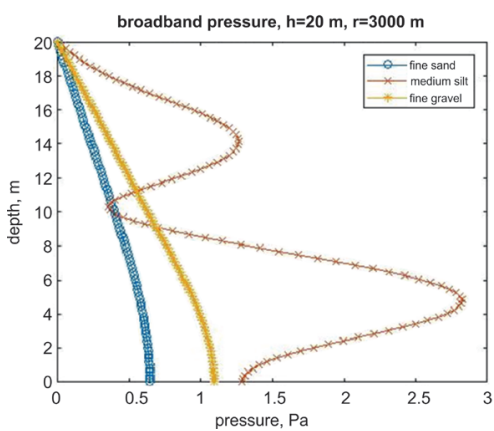

Fig. 16. Vertical distribution of total tug-generated pressure in the distance of $3000 \mathrm{~m}$ from the ship in the case of propagation over the seabed composed of fine sand, medium silt and fine gravel, respectively

The total ship-generated pressure distributions confirm that effects of disturbance propagation in the form of wave modes are visible only up to some distance. The wave propagates further in the form of one mode. The distance depends on a kind of seabed. In the considered case higher modes fade out fastest over fine sand seabed and latest over medium silt seabed. The water layer constitutes a kind of natural low-pass filter for a broad-band signal. Let's monitor changes in the spectrum of underwater acoustic signal produced by the tug in function of distance, in the case of fine gravel seabed. The spectrum determined directly under the ship is shown in Fig. 4. The components of the lowest frequencies fade out in a very short distance from the ship. The components of the frequencies lower than $100 \mathrm{~Hz}$ are practically not present already in $200 \mathrm{~m}$ distance from the ship.

The subsequent components of low frequencies fade out gradually along with growing distance, that can be confirmed by comparing the spectrum determined in the distance of $3000 \mathrm{~m}$ from the ship (Fig. 18) with that under the ship (Fig. 4). The underwater noise signal which reaches the distance of $10000 \mathrm{~m}$ (Fig. 19) is practically a sum of the components having frequencies higher than $400 \mathrm{~Hz}$.

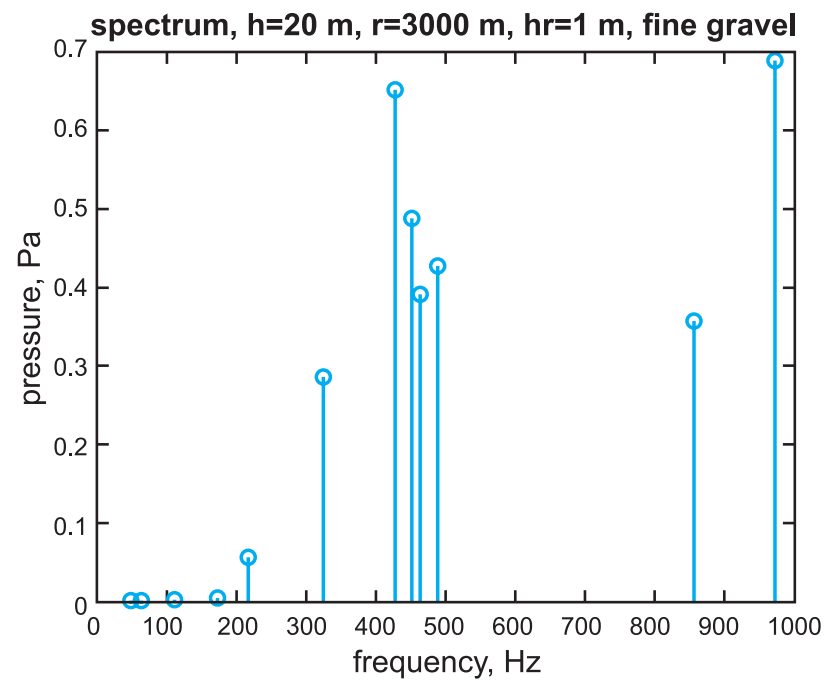

Fig. 18. Underwater noise spectrum of the tug in the distance of $3000 \mathrm{~m}$ from the ship, gravel seabed

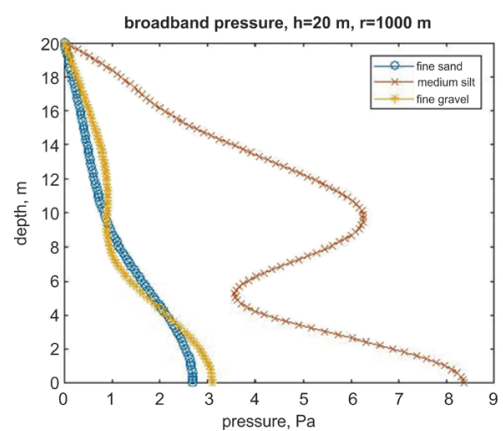

Fig. 17. Vertical distribution of total tug-generated pressure in the distance of $10000 \mathrm{~m}$ from the ship in the case of propagation over the seabed composed of fine sand, medium silt and fine gravel, respectively

\section{FINAL REMARKS}

While assessing the range of acoustic disturbances in the Baltic Sea one should take into account that the acoustic wave propagation in this water area completely differs from the propagation in a deep sea. Application of the spherical propagation model is a too -far -going simplification which may lead to an erroneous estimation of energy transmission losses. The Baltic Sea exemplifies a shallow sea which can be represented as an acoustic system of a flat-parallel waveguide structure filled with liquid layer and seperate boundary conditions for each of the boundaries of the layer.

In this paper the use was made of the method, presented already in the preceding publications, for estimation of energy transmission losses in shallow sea for an idealized case, namely, when free water surface and seabed can be considered planes. The final theoretical result makes it possible to determine the characteristic features of modal propagation of broad-band signal in water areas of different kinds of seabed. The investigations were performed on the basis of these author's own measurements of underwater noise emitted by ships, for definite bottom sediments. Attention was paid to the forming

spectrum, $\mathrm{h}=20 \mathrm{~m}, \mathrm{r}=10000 \mathrm{~m}, \mathrm{hr}=1 \mathrm{~m}$, fine gravel

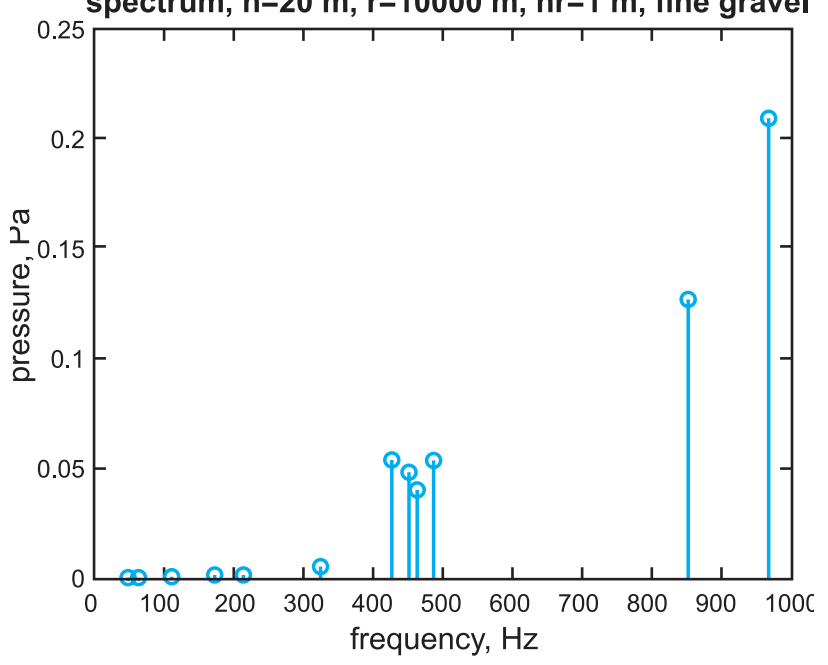

Fig. 19. Underwater noise spectrum of the tug in the distance of $10000 \mathrm{~m}$ from the ship, gravel seabed 
of modes for all frequencies contained in underwater noise spectrum of the considered ship. For the higher spectral frequency components, a greater number of modes is formed and multi-mode propagation phenomenon reaches a greater range than in the case of the low frequency components. A kind of seabed, i.e. its geo-acoustic parameters, affects the range of the total noise generated by the ship, similarly - the range of the propagation in the form of wave modes, that results from that a part of acoustic energy penetrates into seabed.

Attention was drawn to the fact that the water layer has discriminative features which make propagation of the waves having length fourfold greater than water depth, impossible in the conditions assumed in the considered case.

\section{ACKNOWLEDGEMENTS}

The investigations have been performed partly due to funds granted by Polish Ministry of Science and Higher Education for statutory activity of the Faculty of Offshore Engineering and Ship Technology, Gdansk University of Technology, as well as for statutory activity of the Faculty of Navigation and Naval Weapons, Naval Academy in Gdynia.

\section{REFERENCES}

1. Bobkowska K., Janowski A., Jasinska K., Kowal P., Przyborski M.: Light pollution in the context of threats to the wildlife corridors, Nano, bio and green - technologies for a sustainable future, Conference proceedings, SGEM 2016, pp. $665-670$.

2. Briechowskich L. M., Waves in layered media, 2nd Edition, Academic Press, New York 1980.

3. Directive 2008/56/EC of the European Parliament and of the Council of 17 June 2008 establishing a framework for community action in the field of marine environmental policy (Marine Strategy Framework Directive), Official Journal of the European Union 25.6.2008, L 164/19-L164/40.

4. Dymarski C., Dymarski P., Żywicki J.: Design and strength calculations of the tripod support structure for offshore power plant, Polish Maritime Research, Vol. 22, 1, pp. 36-46, 2015.

5. Grelowska G., Kozaczka E., Kozaczka S., Szymczak W.: Gdansk Bay seabed sounding and classification of its results, Polish Maritime Research,Vol 20, 3, pp. 45-50, 2013

6. Grelowska G., Kozaczka E., Kozaczka S., Szymczak W.:, Underwater noise generated by a small ship in the shallow sea, Archives on Acoustics, Vol. 38, 3, pp. 351-356, 2013.

7. Grelowska G.: Study of seasonal acoustic properties of sea water in selected waters of the southern Baltic, Polish Maritime Research, Vol. 23, 1, pp. 25-30, 2016.
8. Grelowska G., Kozaczka E.: Underwater acoustic imaging of the sea, Archives of Acoustics, Vol. 39, 4, pp. 439-452, 2014.

9. Hamilton E.L., Bachman R.T.: Sound velocity and related properties of marine sediments, The Journal of the Acoustical Society of America 72, 6, pp. 1891-1906, 1982.

10. Kozaczka E., Gloza I.: Determination of the ship signature in the very shallow water, Proceedings of the $14^{\text {th }}$ International Congress on Sound and Vibration, Cairns, June 25-29, 2007.

11. Kozaczka E.,, Domagalski J., Gloza I.: Investigation of the underwater noise produced by ships by means of intensity method, Polish Maritime Research, Vol. 17, 3, pp. 26-36, 2010.

12. Kozaczka E., Grelowska G., Szymczak W., Kozaczka S.: The examination of the upper layer of the seabed by means of the acoustic methods, Acta Physica Polonica A, Vol. 119, 6A, pp. 1091-1094, 2011.

13. Kozaczka E., Grelowska G.: Theoretical model of acoustic wave propagation in shallow water, Polish Maritime Research, Vol. 24, 2, pp. 48-55, 2017.

14. Kozaczka E.: The propagation of the acoustic disturbances in the shallow water, in Hydroacoustics of Shallow Water, eds. E. Kozaczka and G. Grelowska, Institute of Fundamental Technological Research, Polish Academy of Sciences, Warszawa, 2013, pp. 31-52.

15. Kozaczka E., Grelowska G.: Shipping low frequency noise and its propagation in shallow water, Acta Physica Polonica A, Vol. 119, 6A, pp. 1009-1012, 2011.

16. McKenna M. F., Ross D., Wiggins S. M., Hildebrand J. A.: Underwater radiated noise from modern commercial ships, J. Acoust. Soc. Am. Vol. 131, 1, pp. 92-103, 2012.

17. Kuperman W. A., Lynch J. F.: Shallow-Water Acoustics, Physics Today, October 2004, pp. 55-61

18. MSFD Technical Subgroup on Underwater Noise: Monitoring Guidance for Underwater Noise in European Seas, Report EUR 26557 EN, European Commission 2014.

19. Tęgowski J.: Acoustic classification of bottom sediments (in Polish), Rozprawy i monografie (Treatises and monographs), Oceanology Institute, Polish Academy of Sciences, Sopot 2006.

20. Weryk M., Kozaczka E., Grelowska G.: Study of noise propagation for small vessels, Archives of Acoustics, Vol. 40, 2 , pp. 267-272, 2015. 


\section{CONTACT WITH THE AUTHOR}

\section{Eugeniusz Kozaczka}

e-mail:kozaczka@pg.edu.pl

Gdańsk University of Technology

Faculty of Ocean Engineering and Ship Technology

Department of Hydromechanics and Hydroacoustics

Narutowicza 11/12, 80-233 Gdansk,

Polish Naval Academy

Faculty of Navigation and Naval Weapons

Department of Hydroacoustics

ul. Śmidowicza 69, 81-127 Gdynia

\section{Poland}

\section{Grażyna Grelowska}

e-mail: grazyna.grelowska@pg.gda.pl

Gdańsk University of Technology

Faculty of Ocean Engineering and Ship Technology

Department of Hydromechanics and Hydroacoustics

Narutowicza 11/12, 80-233 Gdansk

Poland 